\title{
Neutropenic Enterocolitis Associated with Methotrexate Therapy for Rheumatoid Arthritis: A Case Report
}

\author{
Yeong-Jang Lin *, Hung-An Chen and Pei-Chih Chen
}

Division of Allergy, Immunology and Rheumatology, Chi-Mei Medical Center, Tainan, Taiwan

"Corresponding author: Yeong-Jang Lin, Division of Allergy, Immunology and Rheumatology, Chi-Mei Medical Center, No.901, Zhonghua Rd., Yongkang Dist., Tainan City 710, Taiwan, Tel: +886-6-2812811; Fax: +886-6-2828928; E-mail: linyeongjang@yahoo.com.tw

Received: 03 April 2014, Accepted: 02 May 2014, Pub date: 09 May 2014

Copyright: ( 2014 Lin YJ, et al. This is an open-access article distributed under the terms of the Creative Commons Attribution License, which permits unrestricted use, distribution, and reproduction in any medium, provided the original author and source are credited.

\begin{abstract}
Neutropenic enterocolitis is a rare, life-threatening disease, characterized by fever and abdominal pain in the setting of neutropenia. It occurs most commonly in oncologic patients who are profoundly neutropenic as a result of cytotoxic chemotherapy. Neutropenic enterocolitis as a complication of medical therapy for rheumatoid arthritis (RA) has been rarely reported in literature. We describe a patient with RA who developed neutropenic enterocolitis as a complication of methotrexate therapy.
\end{abstract}

Keywords: Methotrexate; Neutropenic enterocolitis; Rheumatoid arthritis

\section{Introduction}

Neutropenic enterocolitis is a rare, life-threatening disease, characterized by a transmural inflammation of the bowel wall, which may progress to perforation and sepsis, in patients with severe neutropenia [1]. It occurs most commonly in oncologic patients who are profoundly neutropenic as a result of cytotoxic chemotherapy. Some non-oncologic conditions have also been associated with neutropenic enterocolitis, including myelodysplastic syndrome, aplastic anemia, acquired immunodeficiency syndrome, cyclic or drug-induced neutropenia, and immunosuppressive therapy for transplants [2]. Neutropenic enterocolitis is a rare complication of medical therapy for rheumatoid arthritis (RA), and only one case has been reported in English literature [3]. Here we describe a case of RA who developed neutropenic enterocolitis associated with methotrexate (MTX) therapy.

\section{Case Report}

An 80-year-old woman had seropositive RA with multiple joint deformities for more than 20 years. Besides, she had hypertension and chronic kidney disease stage 3 . She was treated with prednisolone $5 \mathrm{mg}$ daily, MTX 7.5 mg once a week and folic acid $5 \mathrm{mg}$ daily, and achieved a low disease activity state. She presented to our emergency room with a 10 -day history of sorethroat and dysphagia. Fever $\left(38.8^{\circ} \mathrm{C}\right)$ was noted on arrival. Physical examination revealed severe mucositis with oral candidiasis. Her laboratory findings were as follows: white blood cell (WBC) count $900 / \mathrm{mm}^{3}$ with an absolute neutrophil count $279 / \mathrm{mm}^{3}$, hemoglobin $5.5 \mathrm{gm} / \mathrm{dl}$, platelet count $59,000 / \mathrm{mm}^{3}$, C-reactive protein $28 \mathrm{mg} / \mathrm{L}$ (normal < 6) and creatinine $3.5 \mathrm{mg} / \mathrm{dl}$ (normal 0.6-1.3). She was admitted under the impression of MTX-induced myelosuppression with neutropenic fever. Abdominal pain occurred on the 3rd day of hospitalization. Abdominal examination revealed normoactive bowel sounds, tenderness and rebound tenderness in the right lower quadrant. Obturator and iliopsoas signs were negative. Non-contrast-enhanced computed tomography (CT) of the abdomen demonstrated circumferential wall thickening of cecum and ascending colon with pericolic infiltration (Figure 1), supporting the diagnosis of neutropenic enterocolitis. The patient improved gradually with nonoperative management including total parenteral nutrition with bowel rest, blood product support, granulocyte-colony stimulating factor, leucovorin supplements, and broad-spectrum antibiotic therapy. On the 12th day of hospitalization, her abdominal pain resolved and the WBC count also returned to normal. After discharge, she was treated with hydroxychloroquine $200 \mathrm{mg}$ once daily and sulfasalazine $500 \mathrm{mg}$ twice daily, in combination with prednisolone $5 \mathrm{mg}$ daily. She achieved a state of low disease activity with current therapy.

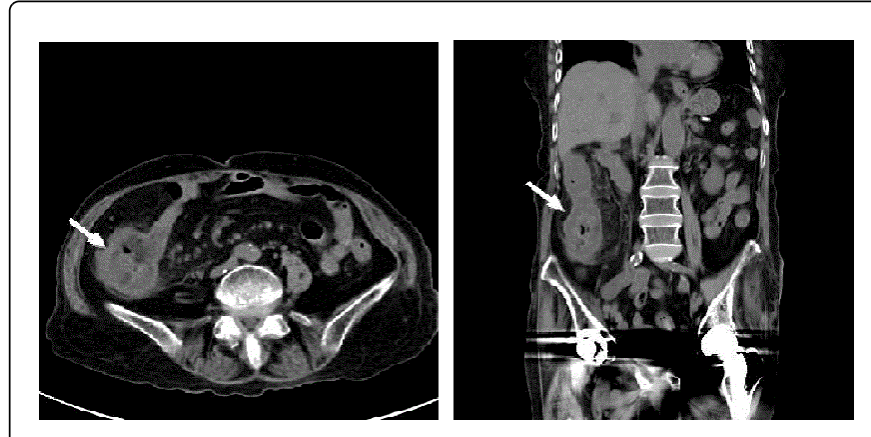

Figure 1: Abdominal non-enhanced CT images (A) Transverse section at the level of ileocecal junction shows circumferential wall thickening of cecum with submucosal edematous change and pericolic infiltration (arrow). (B) Coronal section shows circumferential bowel wall thickening from cecum to ascending colon (arrow).

\section{Discussion}

The exact pathogenesis of neutropenic enterocolitis is incompletely understood. It is thought to result from intestinal mucosal damage caused by cytotoxic drugs or neutropenia itself, followed by unimpeded microbial invasion of the bowel wall in the setting of neutropenia, leading to bowel wall inflammation, edema, necrosis, and 
Page 2 of 2

perforation [4]. Neutropenic enterocolitis may involve nearly any segment of the gastrointestinal tract, with a predilection for the ileocecal area. Patient typically presented with fever, neutropenia and right lower quadrant abdominal pain, which may mimic acute appendicitis. CT scan is the diagnostic modality of choice that can help differentiate neutropenic enterocolitis from appendicitis, diverticulitis, abscess, and Clostridium difficile colitis. Common CT findings include colonic wall thickening, intramural edema or hemorrhage, pericolic inflammation, and pneumatosis intestinalis [5].

The optimal therapy of neutropenic enterocolitis remains controversial due to a paucity of high-quality research studies. Initial non-operative management with adequate fluid resuscitation, bowel rest, nutritional support, and broad-spectrum antibiotic therapy is recommended. Surgery is reserved for patients with bowel obstruction, necrosis, perforation, severe bleeding, or clinical deterioration despite aggressive medical and supportive therapy [2]. Early detection of neutropenic enterocolitis is important to improved patient outcomes, and permits most cases to recover with non-operative management only.

Neutropenic enterocolitis is a rare complication of medical therapy for RA, and only one case has been reported in English literature. Chakravarty et al. described the first case of neutropenic enterocolitis in a 69-year-old man with RA following sulphasalazine therapy [3]. Patient underwent total proctocolectomy for uncontrolled gastrointestinal bleeding, but developed recurred bleeding with shock and died shortly after the operation. Our case had severe pancytopenia and neutropenic enterocolitis secondary to low-dose MTX therapy for RA. Pancytopenia is an uncommon but potentially fatal complication of low-dose MTX therapy that may occur at any time during therapy without any warning signs. Associated risk factors include renal dysfunction, advanced age, hypoalbuminemia, low serum folate level, and concurrent use of nonsteroidal anti-inflammatory drugs [6]. Among these, renal dysfunction appears to be the most important risk factor for myelosuppression. MTX is primarily excreted via the kidney, through glomerular filtration and proximal tubular secretion. In the face of renal impairment, a reduced excretion of MTX and subsequent serum accumulation may induce bone marrow suppression or other toxicities. However, there are no data to guide dose adjustment of MTX in patients with renal impairment, and it has been recommended that low-dose MTX should not be prescribed if the estimated creatinine clearance rate is less than $30 \mathrm{ml} / \mathrm{min}$ [7].

\section{Conclusion}

Neutropenic enterocolitis is a rare but life-threatening complication of low-dose MTX therapy for RA in the setting of severe kidney disease or injury. It is important to consider the possibility of this disease entity in patients with neutropenia from any causes, not only in oncologic patients. A high index of suspicion is important for an early diagnosis, prompt appropriate therapy and improved patient outcomes.

\section{References}

1. Ullery BW, Pieracci FM, Rodney JR, Barie PS (2009) Neutropenic enterocolitis. Surg Infect (Larchmt) 10: 307-314.

2. Machado NO (2010) Neutropenic enterocolitis: A continuing medical and surgical challenge. N Am J Med Sci 2: 293-300.

3. Chakravarty K, Scott DG, McCann BG (1992) Fatal neutropenic enterocolitis associated with sulphasalazine therapy for rheumatoid arthritis. Br J Rheumatol 31: 351-353.

4. Urbach DR, Rotstein OD (1999) Typhlitis. Can J Surg 42: 415-419.

5. Vas WG1, Seelig R, Mahanta B, Patel B, Salimi Z, et al. (1988) Neutropenic colitis: evaluation with computed tomography. J Comput Tomogr 12: 211-215.

6. Gutierrez-Ureña S, Molina JF, García CO, Cuéllar ML, Espinoza LR (1996) Pancytopenia secondary to methotrexate therapy in rheumatoid arthritis. Arthritis Rheum 39: 272-276.

7. Saag KG, Teng GG, Patkar NM, Anuntiyo J, Finney C, et al. (2008) American College of Rheumatology 2008 recommendations for the use of nonbiologic and biologic disease-modifying antirheumatic drugs in rheumatoid arthritis. Arthritis Rheum 59: 762-784. 